\title{
Yukawa-unified natural supersymmetry
}

\author{
Howard Baer, ${ }^{a}$ Sabine Kraml ${ }^{b}$ and Suchita Kulkarni ${ }^{b}$ \\ ${ }^{a}$ Department of Physics and Astronomy, University of Oklahoma, \\ Norman, OK 73019, U.S.A. \\ ${ }^{b}$ Laboratoire de Physique Subatomique et de Cosmologie, UJF Grenoble 1, CNRS/IN2P3, INPG, \\ 53 Avenue des Martyrs, F-38026 Grenoble, France \\ E-mail: baer@nhn.ou.edu, sabine.kraml@lpsc.in2p3.fr, \\ suchita.kulkarni@lpsc.in2p3.fr
}

Abstract: Previous work on $t-b-\tau$ Yukawa-unified supersymmetry, as expected from SUSY GUT theories based on the gauge group $\mathrm{SO}(10)$, tended to have exceedingly large electroweak fine-tuning (EWFT). Here, we examine supersymmetric models where we simultaneously require low EWFT ("natural SUSY") and a high degree of Yukawa coupling unification, along with a light Higgs scalar with $m_{h} \sim 125 \mathrm{GeV}$. As Yukawa unification requires large $\tan \beta \sim 50$, while EWFT requires rather light third generation squarks and low $\mu \approx 100-250 \mathrm{GeV}, B$-physics constraints from $\operatorname{BR}\left(B \rightarrow X_{s} \gamma\right)$ and $\operatorname{BR}\left(B_{s} \rightarrow \mu^{+} \mu^{-}\right)$ can be severe. We are able to find models with EWFT $\Delta \lesssim 50-100$ (better than 1-2\% EWFT) and with Yukawa unification as low as $R_{\text {yuk }} \sim 1.2$ (20\% unification). The unification is lessened to $R_{\text {yuk }} \sim 1.3$ when $B$-physics constraints are imposed. We present several Yukawa-unified natural SUSY (YUNS) benchmark points. LHC searches will be able to access gluinos in the lower $1-2 \mathrm{TeV}$ portion of their predicted mass range although much of YUNS parameter space may lie beyond LHC14 reach. If heavy Higgs bosons can be accessed at a high rate, then the rare $H, A \rightarrow \mu^{+} \mu^{-}$decay might allow a determination of $\tan \beta \sim 50$ as predicted by YUNS models. Finally, the predicted light higgsinos should be accessible to a linear $e^{+} e^{-}$collider with $\sqrt{s} \sim 0.5 \mathrm{TeV}$.

KEYWords: Supersymmetry Phenomenology

ARXiv EPRINT: 1208.3039 


\section{Contents}

1 Introduction 1

2 Parameter space and Yukawa unification 4

$\begin{array}{lll}3 & \text { Scan results } & 6\end{array}$

$\begin{array}{lll}4 & \text { Benchmark points } & 10\end{array}$

5 Yukawa-unified natural SUSY: LHC, ILC and DM searches 12

$\begin{array}{lll}5.1 & \text { YUNS at LHC } & 12\end{array}$

5.2 YUNS at ILC 13

$\begin{array}{lll}5.3 & \text { Higgsino-like WIMPs } & 13\end{array}$

6 Summary and conclusions $\quad 14$

\section{Introduction}

A striking feature in nature is that all the fermions of each generation fill out a complete 16-dimensional spinor multiplet of the gauge group $\mathrm{SO}(10)[1-5]$. While ordinary grand unified theories (GUTs) suffer from the notorious gauge hierarchy problem, supersymmetric (SUSY) GUTs not only tame this hierarchy problem $[6,7]$, but they also receive support from the well-known unification of gauge couplings [8-13]. In the simplest $\mathrm{SO}(10)$ SUSY GUT theories, where both MSSM Higgs doublets $H_{u}$ and $H_{d}$ occupy the same 10-dimensional representation, one also expects unification of third generation Yukawa couplings $f_{t}, f_{b}$ and $f_{\tau}$ at $M_{\mathrm{GUT}} \simeq 2 \times 10^{16} \mathrm{GeV}$ [14-37]. The $t-b-\tau$ Yukawa coupling unification is highly sensitive to both 2-loop renormalization group running (RGEs) and to threshold corrections when transitioning between MSSM and SM effective theories at the SUSY particle mass scale. Thus, the entire SUSY mass spectrum enters into a precise computation of Yukawa coupling unification.

Many groups have explored $t-b-\tau$ Yukawa unification (YU) in SUSY theories [38-50]. It has been found that, for $\mu>0$, YU can occur at the few percent level in either the Higgs splitting (HS) model or the DR3 model (D-term splitting, right-hand neutrino effects and third generation splitting), provided that the GUT scale soft SUSY breaking (SSB) terms are related as

$$
A_{0}^{2} \simeq 2 m_{10}^{2} \simeq 4 m_{16}^{2}
$$

with $A_{0}<0$. Moreover, the GUT scale Higgs splitting $m_{H_{u}}^{2}<m_{H_{d}}^{2}$ is needed to allow for an appropriate radiative breakdown of electroweak symmetry. In these models, first generation squarks and sleptons are required to be in the multi- $\mathrm{TeV}$ range $[41,46]$ 
while third generation sfermions are driven to much lighter TeV-scale masses. A benefit of these models is that the light SUSY Higgs boson mass $m_{h}$ tends naturally to be in the $125 \mathrm{GeV}$ range, ${ }^{1}$ as required by the recent LHC discovery [53, 54]. The gaugino masses from YU SUSY are expected to be quite light, with typically $m_{\tilde{g}} \lesssim 500 \mathrm{GeV}$ (now excluded by LHC searches for gluino pair production along with cascade decay into states containing $b$-quarks [55]), although solutions with heavier gluinos $\sim 1-2 \mathrm{TeV}$ can also be found [56]. In all these cases, the superpotential $\mu$ parameter, which is extracted from the electroweak minimization conditions, occupies values in the $1-10 \mathrm{TeV}$ regime, leading to severe electroweak fine-tuning (EWFT).

In this work, we examine to what extent it is possible to reconcile YU with low EWFT. Minimization of the SUSY scalar potential allows one to relate the $Z$ mass scale to the superpartner mass scale via the well-known relation

$$
\frac{1}{2} M_{Z}^{2}=\frac{\left(m_{H_{d}}^{2}+\Sigma_{d}\right)-\left(m_{H_{u}}^{2}+\Sigma_{u}\right) \tan ^{2} \beta}{\left(\tan ^{2} \beta-1\right)}-\mu^{2} .
$$

The radiative corrections $\Sigma_{u}$ and $\Sigma_{d}$ are given in the 1-loop approximation of the Higgs effective potential by:

$$
\Sigma_{u, d}=\frac{1}{v_{u, d}} \frac{\partial \Delta V}{\partial H_{u, d}}
$$

where $\Delta V$ is the one-loop correction to the tree-level potential, and the derivative is evaluated in the physical vacuum: i.e. the fields are set to their vacuum expectation values after evaluating the derivative. At the one-loop level and in the limit of setting first/second generation Yukawa couplings to zero, $\Sigma_{u}$ contains 18 and $\Sigma_{d}$ contains 19 separate contributions from various particles/sparticles [57]. We include contributions from $W^{ \pm}, Z, \tilde{t}_{1,2}$, $\tilde{b}_{1,2}, \tilde{\tau}_{1,2}, \widetilde{W}_{1,2}, \widetilde{Z}_{1,2,3,4}, t, b$ and $\tau, h, H$ and $H^{ \pm}$. We adopt a scale choice $Q^{2}=m_{\tilde{t}_{1}} m_{\tilde{t}_{2}}$ to minimize the largest of the logarithms. The dominant contribution to the terms $\Sigma_{u, d}$ arise from superpotential Yukawa interactions of third generation squarks involving the top quark Yukawa coupling. For instance, the dominant contribution to $\Sigma_{u}$ is given by

$$
\Sigma_{u}\left(\tilde{t}_{1,2}\right)=\frac{3}{16 \pi^{2}} F\left(m_{\tilde{t}_{1,2}}^{2}\right)\left[f_{t}^{2}-g_{Z}^{2} \mp \frac{f_{t}^{2} A_{t}^{2}-8 g_{Z}^{2}\left(\frac{1}{4}-\frac{2}{3} x_{W}\right) \Delta_{t}}{m_{\tilde{t}_{2}}^{2}-m_{\tilde{t}_{1}}^{2}}\right],
$$

where $\Delta_{t}=\left(m_{\tilde{t}_{L}}^{2}-m_{\tilde{t}_{R}}^{2}\right) / 2+m_{Z}^{2} \cos 2 \beta\left(\frac{1}{4}-\frac{2}{3} x_{W}\right), g_{Z}^{2}=\left(g^{2}+g^{\prime 2}\right) / 8$ and $x_{W} \equiv \sin ^{2} \theta_{W}$ and $F\left(m^{2}\right)=m^{2}\left(\log \frac{m^{2}}{Q^{2}}-1\right)$. This expression thus grows quadratically with the stop mass.

We adopt the fine-tuning measure from [57], which requires that each of the 40 terms on the right-hand-side (r.h.s. ) of eq. (1.2) should be of order $\sim m_{Z}^{2} / 2$. Labeling each term as $C_{i}$ (with $i=H_{d}, H_{u}, \mu, \Sigma_{d}^{d}\left(\tilde{t}_{1}\right), \Sigma_{u}^{u}\left(\tilde{t}_{1}\right)$, etc.), we may require $C_{\max } \equiv \max \left|C_{i}\right|<$ $\Lambda_{\max }^{2}$, where $\Lambda_{\max } \sim 100-300 \mathrm{GeV}$, depending on how much EWFT one is willing to tolerate. This measure of fine-tuning is similar to (but not exactly the same as) KitanoNomura [58, 59] but different from Barbieri-Giudice [60] beyond the tree-level. In the

\footnotetext{
${ }^{1}$ The large negative $A_{0}$ in eq. (1.1) leads to maximal stop mixing, see e.g. [51, 52], thus increasing $m_{h}$ to the desired range.
} 
following, we will use the fine-tuning parameter

$$
\Delta=C_{\max } /\left(m_{Z}^{2} / 2\right)
$$

where lower values of $\Delta$ correspond to less fine-tuning, and e.g. $\Delta=20$ would correspond to $\Delta^{-1}=5 \%$ fine-tuning.

Our goal in this paper is to search for parameter choices which

1. Maximize the degree of Yukawa coupling unification, i.e. minimize $R_{\mathrm{yuk}}=$ $\max \left(f_{t}, f_{b}, f_{\tau}\right) / \min \left(f_{t}, f_{b}, f_{\tau}\right)$ with each Yukawa coupling evaluated at the GUT scale. Thus, a value of $R_{\text {yuk }}=1$ would give perfect Yukawa coupling unification.

2. Have as low EWFT as possible (in practice, we will require $\Delta \lesssim 100$, or better than $1 \%$ EWFT).

3. Have $m_{h} \approx 125 \mathrm{GeV}$ in accord with the recent LHC discovery of a Higgs-like resonance. In practice, we will require $122 \mathrm{GeV}<m_{h}<128 \mathrm{GeV}$ to allow for a roughly $2-3 \mathrm{GeV}$ error in the RG-improved one-loop effective potential calculation of the Higgs mass $m_{h}$.

We recognize that in addition to EWFT, there also exists a fine-tuning associated with generating particular weak scale SUSY spectra from distinct GUT scale parameters [60], (see also [61, 62] for related discussions). Here we adopt the less restrictive weak scale finetuning condition, which nonetheless turns out to be indeed very restrictive. In this vein, we regard particular GUT scale parameters as merely a parametrization of our ignorance of the mechanism of SUSY breaking and soft term generation. ${ }^{2}$ For instance, in this paper we will require rather low values of superpotential $\mu$ parameter to avoid excessive EWFT in eq. (1.2). In generic SUSY models, the value of $\mu$ is expected to be of order $M_{\text {Planck }}$ since it is a dimensionful SUSY-preserving parameter. Excessively large $\mu$ can be avoided ala Giudice-Masiero [65] where the $\mu$ superpotential term is forbidden by some high scale symmetry, but then is regenerated as a soft SUSY breaking term via a Higgs-Higgs coupling to the hidden sector.

For the remainder of this paper, in section 2 we present details of our scan over SUSY parameter space, and which constraints are invoked in our analysis. In section 3, we present the results of our parameter space scans. We will find that requiring $\Delta \lesssim 100$ and $m_{h} \sim 125 \mathrm{GeV}$ allows for $R_{\text {yuk }} \lesssim 1.3$ once all applicable constraints are taken into account. While this degree of Yukawa unification is not optimal, we feel it is still useful in that it might guide model builders towards models including additional GUT scale threshold corrections or extra matter or above-GUT-scale running which may ameliorate the situation. In section 5, we discuss observable consequences of YUNS for LHC, ILC and dark matter searches. We pay some attention to methods which might allow one to distinguish YUNS from generic NS models at lower $\tan \beta$ values. In section 6 we present a summary and conclusions.

\footnotetext{
${ }^{2}$ The relation between GUT scale fine-tuning and the $b-\tau$ Yukawa coupling ratio was studied in [63, 64].
} 


\section{Parameter space and Yukawa unification}

For our calculations, we adopt the ISAJET 7.83 [66] SUSY spectrum generator ISASUGRA [67, 68]. ISASUGRA begins the calculation of the sparticle mass spectrum with input $\overline{D R}$ gauge couplings and $f_{b}, f_{\tau}$ Yukawa couplings at the scale $Q=M_{Z}\left(f_{t}\right.$ running begins at $Q=m_{t}$ ) and evolves the 6 couplings up in energy to scale $Q=M_{\mathrm{GUT}}$ (defined as the value $Q$ where $g_{1}=g_{2}$ ) using two-loop RGEs. At $Q=M_{\mathrm{GUT}}$, we input the soft SUSY breaking parameters as boundary conditions, and evolve the set of 26 coupled MSSM RGEs [69] back down in scale to $Q=M_{Z}$. Full two-loop MSSM RGEs are used for soft term evolution, while the gauge and Yukawa coupling evolution includes threshold effects in the one-loop beta-functions, so the gauge and Yukawa couplings transition smoothly from the MSSM to SM effective theories as different mass thresholds are passed. In ISASUGRA, the values of SSB terms of sparticles which mix are frozen out at the scale $Q \equiv M_{\mathrm{SUSY}}=\sqrt{m_{\tilde{t}_{L}} m_{\tilde{t}_{R}}}$, while non-mixing SSB terms are frozen out at their own mass scale $[67,68]$. The scalar potential is minimized using the RG-improved one-loop MSSM effective potential evaluated at an optimized scale $Q=M_{\text {SUSY }}$ which accounts for leading two-loop effects [70]. Once the tree-level sparticle mass spectrum is computed, full one-loop radiative corrections are calculated for all sparticle and Higgs boson masses, including complete one-loop weak scale threshold corrections for the top, bottom and tau masses at scale $Q=M_{\text {SUSY }}[71-74]$. Since the GUT scale Yukawa couplings are modified by the threshold corrections, the ISAJET RGE solution must be imposed iteratively with successive updown running until a convergent sparticle mass solution is found. Since ISASUGRA uses a "tower of effective theories" approach to RG evolution, we expect a more accurate evaluation of the sparticle mass spectrum for models with split spectra (this procedure sums the logarithms of potentially large ratios of sparticle masses) than with programs which make an all-at-once transition from the MSSM to SM effective theories. The fine-tuning measure $\Delta$ described in section 1 has been implemented in ISAJET 7.83 [66].

In models of "natural SUSY" (NS) [57-59, 75-81], the first requirement to gain a low EWFT is that the $\mu$ parameter be of the order of $\sim M_{Z}$, while in Yukawa-unified SUSY, it is necessary to invoke some manner of Higgs soft term splitting at the GUT scale in order to obtain raditive EWSB. For these reasons, the model parameter space chosen in this study is the two-parameter non-universal Higgs model (NUHM2) where the weak scale values of $\mu$ and $m_{A}$ are input in lieu of the GUT scale values of $m_{H_{u}}^{2}$ and $m_{H_{d}}^{2}$. In addition, to allow for (sub)TeV-scale third generation masses (as required by EWFT from eq. (1.2) along with at least a partial decoupling solution to the SUSY flavor and CP problems, we allow for split first/second and third generations at the GUT scale. Thus, the parameter space we choose is given by

$$
m_{16}(1,2), m_{16}(3), m_{1 / 2}, A_{0}, \tan \beta, \mu, m_{A} .
$$

Here, $m_{16}(1,2)$ and $m_{16}(3)$ are the first/second and third generation sfermion soft masses, respectively; $m_{1 / 2} \equiv M_{1}=M_{2}=M_{3}$ is the universal gaugino mass parameter; and $A_{0} \equiv A_{t}=A_{b}=A_{\tau}$ is the universal trilinear coupling. These parameters are defined at 
$M_{\mathrm{GUT}}$, while $\tan \beta, \mu$, and $m_{A}$ are defined at the weak scale. The top quark mass is set to $m_{t}=173.2 \mathrm{GeV}$.

We search for mass spectra with low EWFT $\Delta$ and low $R_{\text {yuk }}$ by performing a vast random scan over the following parameter ranges (masses in $\mathrm{GeV}$ units):

$$
\begin{aligned}
5000 \mathrm{GeV} & <m_{16}(1,2)<20000 \mathrm{GeV} \\
0 & <m_{16}(3)<20000 \mathrm{GeV} \\
300 \mathrm{GeV} & <m_{1 / 2}<2000 \mathrm{GeV} \\
-2 & <A_{0} / m_{16}(3)<1 \\
50 & <\tan \beta<60 \\
100 \mathrm{GeV} & <\mu<250 \mathrm{GeV} \\
500 \mathrm{GeV} & <m_{A}<5000 \mathrm{GeV}
\end{aligned}
$$

The lower limit on $m_{1 / 2}$ comes from the approximate LHC bound of $m_{\tilde{g}} \gtrsim 900 \mathrm{GeV}$ (for $\left.m_{\tilde{q}} \gg m_{\tilde{g}}\right)[82,83]$, while the lower bound on $m_{A}$ comes from LHC searches for $A, H \rightarrow$ $\tau^{+} \tau^{-}$which require $m_{A}>500 \mathrm{GeV}$ at $\tan \beta \sim 50$ [84]. We require of our solutions that

1. electroweak symmetry be radiatively broken (REWSB),

2. the neutralino $\widetilde{Z}_{1}$ is the lightest MSSM particle,

3. the light chargino mass obeys the rather model independent LEP2 limit that $m_{\widetilde{W}_{1}}>$ $103.5 \mathrm{GeV}$ [85], and

4. the light Higgs mass falls within the window $m_{h}=122-128 \mathrm{GeV}$, where we adopt $\pm 3 \mathrm{GeV}$ as theoretical error on the Higgs mass calculation.

Regarding $B$-physics constraints, we consider $\operatorname{BR}\left(B \rightarrow X_{s} \gamma\right)=(3.55 \pm 0.34) \times 10^{-4}$, where experimental and SM theoretical uncertainties [86] have been added in quadrature. We combine the above uncertainty with $\mathrm{a} \sim 5 \%$ uncertainty from new physics (SUSY) contributions, ${ }^{3}$ and then impose the $\operatorname{BR}\left(B \rightarrow X_{s} \gamma\right)$ constraint at the $2 \sigma$ level. For the $\operatorname{BR}\left(B_{s} \rightarrow \mu^{+} \mu^{-}\right)$constraint, we assume a theoretical uncertainty of $10 \%$ [87]. We combine this uncertainty linearly with the $95 \%$ CL limit $\mathrm{BR}\left(B_{s} \rightarrow \mu^{+} \mu^{-}\right)<4.2 \times 10^{-9}$. This leads to the the following limits

$$
\begin{aligned}
\operatorname{BR}\left(B \rightarrow X_{s} \gamma\right) & =[2.77,4.33] \times 10^{-4}, \\
\operatorname{BR}\left(B_{s} \rightarrow \mu^{+} \mu^{-}\right) & <4.62 \times 10^{-9},
\end{aligned}
$$

which we will use throughout the numerical analysis.

Regarding neutralino relic density, we remark here that models of natural SUSY contain a higgsino-like lightest neutralino with thermal abundance of typically $\Omega_{\widetilde{Z}_{1}}^{\mathrm{TP}} h^{2} \sim$ $\mathcal{O}\left(10^{-3}-10^{-2}\right)$. This thermal under-abundance can be regarded as a positive feature

\footnotetext{
${ }^{3}$ We have checked our SUSY calculations of $B R(b \rightarrow s \gamma)$ using both IsaTools and SuperISO. Here, we observe deviations of $\sim 5 \%$ in the relevant region of parameter space.
} 


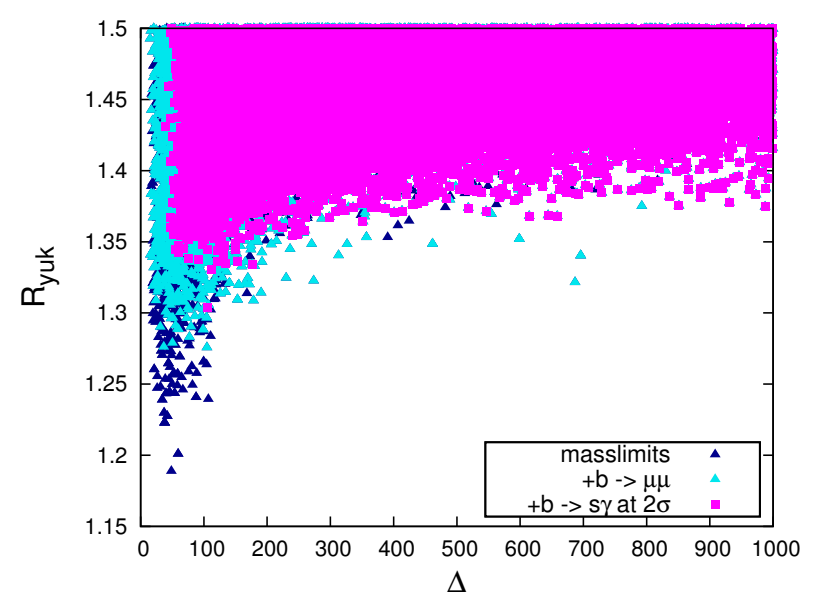

Figure 1. Scatter plot of $R_{\text {yuk }}$ versus $\Delta$ from the scan defined in eq. (2.2) The dark blue triangles violate the $B$-physics constraints of eq. (2.3) The light blue triangles obey the $\operatorname{BR}\left(B_{s} \rightarrow \mu^{+} \mu^{-}\right)$ constraint, but deviate from the measured $\operatorname{BR}\left(B \rightarrow X_{s} \gamma\right)$ by more than $2 \sigma$. Finally, the pink squares satisfy both the $\operatorname{BR}\left(B_{s} \rightarrow \mu^{+} \mu^{-}\right)$and $\operatorname{BR}\left(B \rightarrow X_{s} \gamma\right)$ constraints. Only points with $\Delta<1000$ and $R_{y u k}<1.5$ are shown.

of NS models in the sense that if one invokes the axion solution to the strong CP problem, then one expects mixed axion-higgsino dark matter, where the higgsino portion is typically enhanced by thermal axino production and decay to higgsinos in the early universe. Thus, a thermal under-abundance leaves room for additional non-thermal higgsino production plus an axion component to the dark matter [88].

\section{Scan results}

As our first result, we show in figure 1 points from our parameter space scan in the $R_{\text {yuk }}$ vs. $\Delta$ plane. All points have $m_{h}=122-128 \mathrm{GeV}$ and obey the current LEP and LHC SUSY mass limits. The dark blue triangles however violate the $B$-physics constraints of eq. (2.3). The light blue triangles obey the $\operatorname{BR}\left(B_{s} \rightarrow \mu^{+} \mu^{-}\right)$constraint, but deviate from the measured $\operatorname{BR}\left(B \rightarrow X_{s} \gamma\right)$ by more than $2 \sigma$. Finally, the pink squares satisfy both the $\operatorname{BR}\left(B_{s} \rightarrow \mu^{+} \mu^{-}\right)$and $\operatorname{BR}\left(B \rightarrow X_{s} \gamma\right)$ constraints. This color scheme is used throughout the remainder of the paper. We see already from this plot that flavor physics constraints significantly affect the parameter space of Yukawa-unified natural SUSY. This is to be expected, since naturalness requires lighter third generation squarks while Yukawa unification requires $\tan \beta \sim 50$ : both these effects bolster SUSY contributions to $B$-physics observables. (Analogous observations were made in $[38,39,41,46,89]$ in the context of generic YU models.) Without flavor constrains, we can obtain $R_{\text {yuk }}$ as low as $\sim 1.2$. The $\operatorname{BR}\left(B_{s} \rightarrow \mu^{+} \mu^{-}\right)$constraint pushes this up to $R_{\text {yuk }} \gtrsim 1.27$, and the $\operatorname{BR}\left(B \rightarrow X_{s} \gamma\right)$ constraint to $R_{\text {yuk }} \gtrsim 1.3$. Aside from the $B$-physics constraints, it is intriguing that points with lowest $\Delta$ also have lowest values of $R_{\text {yuk }}$. This is because low $\Delta$ requires light third generation squark masses, while at the same time Yukawa unification requires large SUSY threshold corrections which also require lighter third generation squarks. 

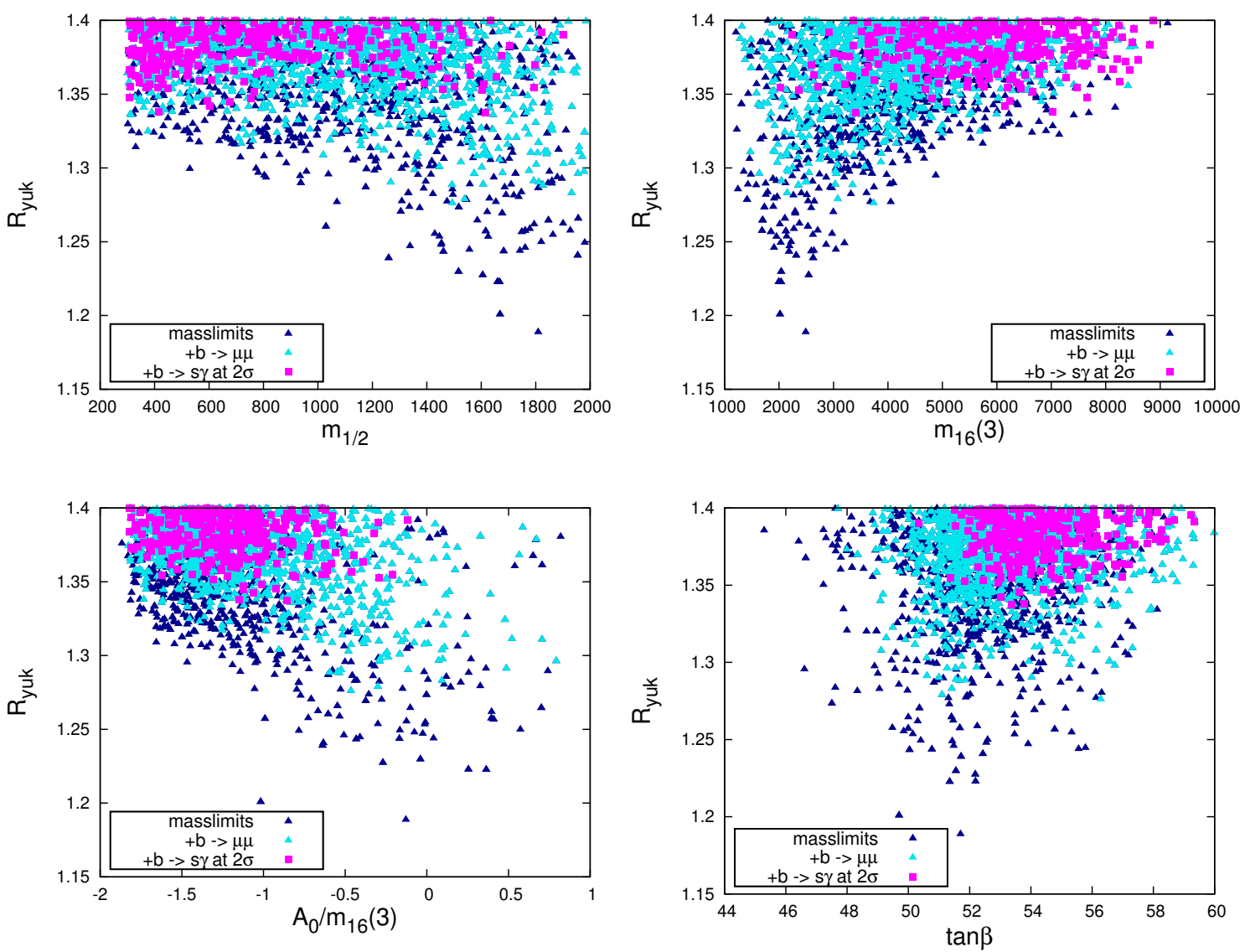

Figure 2. Dependence of $R_{\text {yuk }}$ on $m_{1 / 2}, m_{16}(3), A_{0} / m_{16}(3)$ and $\tan \beta$, for $\Delta<100$. Same color code as in figure 1.

In figure 2 , we show the value of $R_{\text {yuk }}$ versus various input parameters for $\Delta<100$ (less than $1 \%$ fine-tuning). Here, the behavior deviates considerably from $t-b-\tau$ unified models with large $\mu>0$ and a spectrum derived from the radiatively driven inverted scalar mass hierarchy $[40,41,46]$. From frame $a$ ), we see that YUNS models actually prefer large $m_{1 / 2}$ whereas generic Yukawa unified SUSY models (with $R_{\text {yuk }} \lesssim 1.1$ but arbitrary EWFT), YUS for short, prefer low $m_{1 / 2}$. The preference for large $m_{1 / 2}$ helps to avoid LHC constraints on the gluino mass. If we impose the $B$-physics constraints, then the distribution flattens out with some preference for lower $m_{1 / 2}$ values. In frame $b$ ), where $R_{\text {yuk }}$ is plotted $v s$. $m_{16}(3)$, we see that lowest $R_{\text {yuk }}$ values prefer $m_{16}(3) \sim 2 \mathrm{TeV}$, which leads to rather light third generation squarks and typically violation of $B$-physics constraints. If we respect $B$-constraints, then larger values of $m_{16}(3) \sim 3-7 \mathrm{TeV}$ are preferred, at the cost of larger values of $R_{\text {yuk }}$. In frame $c$ ), we plot versus $A_{0} / m_{16}(3)$. For YUS models, there is a strong preference for $A_{0} \sim-2 m_{16}$ [40], while for YUNS, the lowest $R_{\text {yuk values are obtained for }}$ smaller $\left|A_{0}\right|$. Imposing $B$-constraints, the preference moves to $A_{0} \sim(-2$ to 0$) \times m_{16}(3)$. Frame $d$ ) shows $R_{\text {yuk }} v s . \tan \beta$. As expected, $\tan \beta \sim 50$ is preferred.

The dependence of $\Delta$ on the input parameters is shown in figure 3 for points with $R_{\text {yuk }}<1.4$. In frame $a$ ), we see a mild preference by $\Delta$ for low $m_{1 / 2}$, i.e. the gluino 

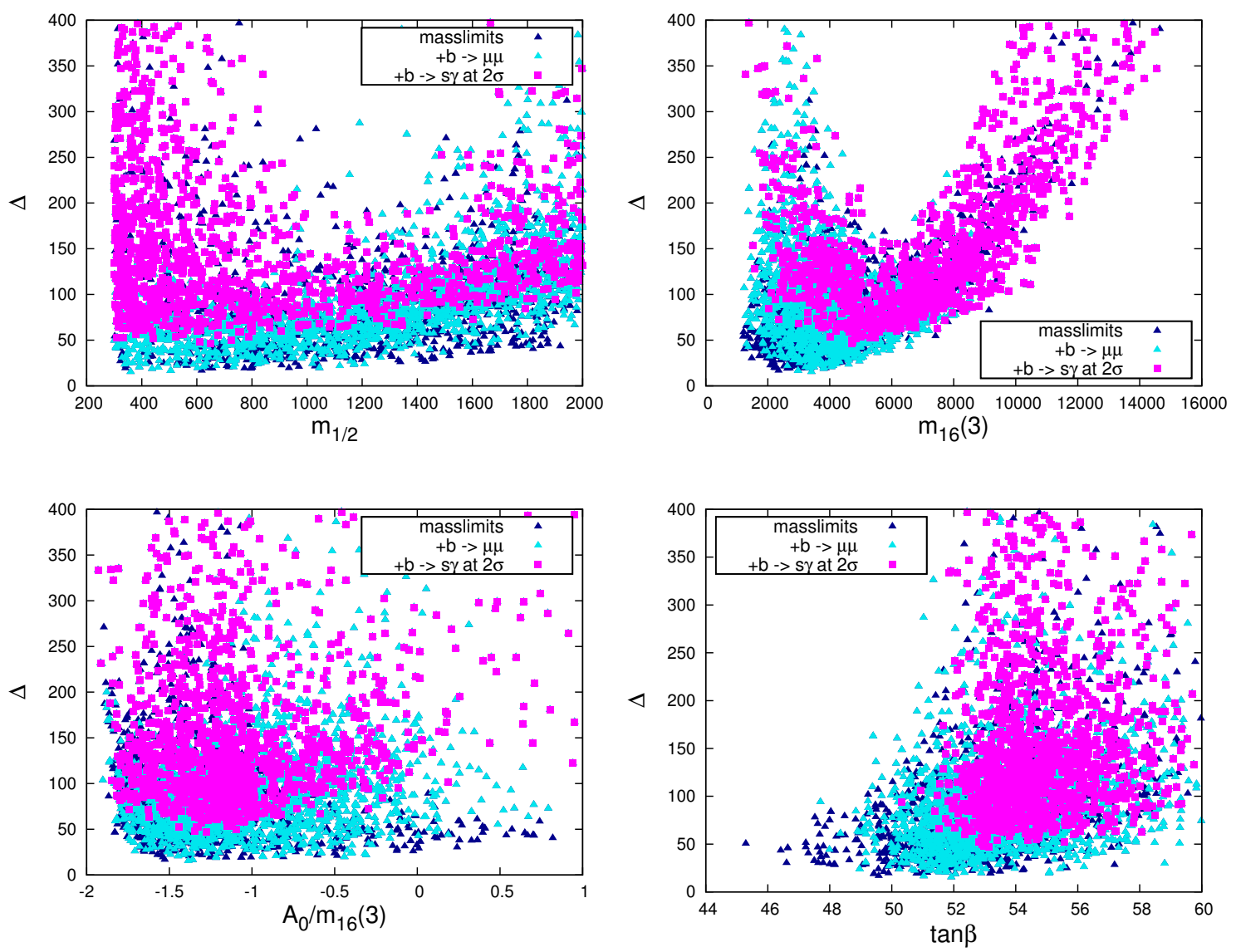

Figure 3. Dependence of fine-tuning $\Delta$ on $m_{1 / 2}, m_{16}(3), A_{0} / m_{16}(3)$ and $\tan \beta$, for $R_{\mathrm{yuk}}<1.4$. Same color code as in figure 1.

mass can't be too heavy, lest it pushes the stop masses too high, leading to large $\Sigma_{u}$. From frame $b$ ), we see low $\Delta$ prefers $m_{16}(3) \sim 2-4 \mathrm{TeV}$. If $m_{16}(3)$ is much higher, then third generation squarks are too heavy to give low EWFT, while if $m_{16}(3)$ is too light, we generate tachyonic spectra: the optimal corresponds to $m_{16}(3) \sim 2-4 \mathrm{TeV}$. For $m_{16}(3) \sim$ $1-2 \mathrm{TeV}$, then sub-TeV top squarks are generated leading to violation of $B$-constraints. In frame $c$ ), we see that low $\Delta$ allows a wide range of $A_{0}$ unless $B$-constraints are imposed, in which case $A_{0} \sim(-2$ to 0$) \times m_{16}(3)$ is again preferred. The large negative $A_{0}$ values lead to larger $m_{h}$ values and also can lower the EWFT [62]. In frame $d$ ), we see that there is only mild preference for $\tan \beta \sim 48-58$ values unless $B$-constraints are respected, in which case $\tan \beta \gtrsim 52$ is preferred.

In figure 4 we show $R_{\text {yuk }}$ versus various sparticle and Higgs masses. In frame $a$ ), the distribution versus $m_{h}$ is shown, and we see that low $R_{\text {yuk }}$ prefers the lower range of $m_{h}$. This is understandable since low EWFT prefers lower top and bottom squark masses, which may not feed a sufficient radiative correction into the $m_{h}$ computation. These low third generation squark mass solutions also tend to give large contributions to $B$-constraint. If we impose $B$-constraints, then $m_{h}$ can live in the $122-128 \mathrm{GeV}$ range, at the cost of larger $R_{\text {yuk }}$. In frame $b$ ), we show the distribution versus $m_{\tilde{g}}$. While all solutions - especially 

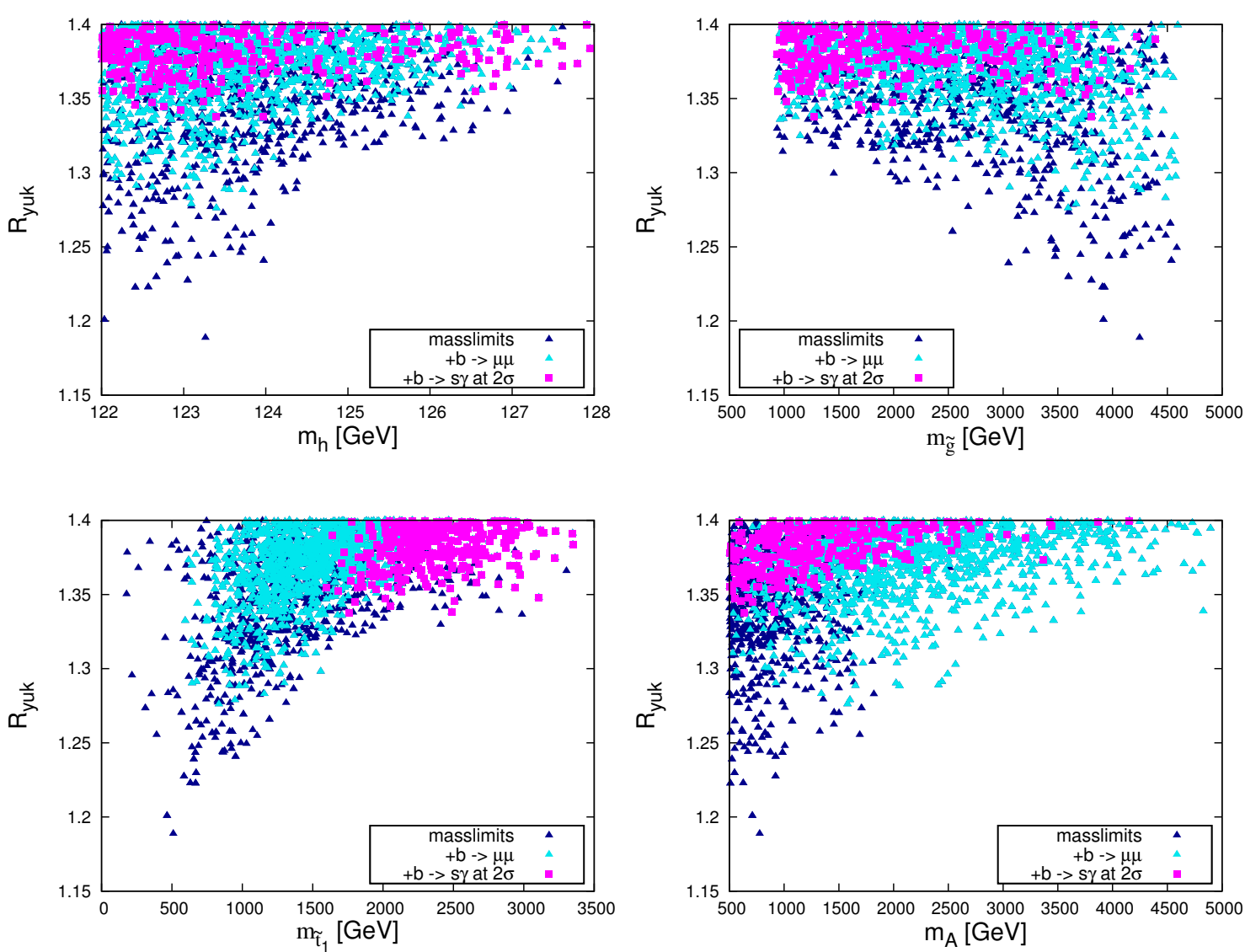

Figure 4. Dependence of $R_{\mathrm{yuk}}$ on $m_{h}, m_{\tilde{g}}, m_{\tilde{t}_{1}}$ and $m_{A}$, for $\Delta<100$. Same color code as in figure 1 .

low $R_{\text {yuk }}$ ones - favor the heavier range of $m_{\tilde{g}}\left(m_{\tilde{g}} \sim 2-5 \mathrm{TeV}\right.$, likely beyond LHC reach), the solutions obeying $B$-constraints tend to slightly favor lower $m_{\tilde{g}}$, possibly within range of LHC14 searches. In frame $c$ ), the distribution versus $m_{\tilde{t}_{1}}$ is shown. Here, we see a clear demarcation: $B$-constraints favor $m_{\tilde{t}_{1}} \gtrsim 1.5 \mathrm{TeV}$ to suppress SUSY loop contributions to $\operatorname{BR}\left(B \rightarrow X_{s} \gamma\right)$. This constraint forces the minimum $R_{\text {yuk }}$ to move from $\sim 1.18$ to about 1.3. (Note that additional small flavor-violating contributions to the MSSM Lagrangian could alter the predicted $\mathrm{BR}\left(B \rightarrow X_{s} \gamma\right)$ and/or $\mathrm{BR}\left(B_{s} \rightarrow \mu^{+} \mu^{-}\right)$rates [101, 102].) In frame $d$ ), we show the distribution versus pseudoscalar. Higgs mass $m_{A}$. We see low $R_{\text {yuk }}$ favors the lower range of $m_{A}$, although values up to and beyond $5 \mathrm{TeV}$ are also possible (at the cost of $R_{\text {yuk }} \sim 1.4$, however). Since $\tan \beta \sim 50$, LHC searches for $A, H \rightarrow \tau^{+} \tau^{-}$ will access a significant range of $m_{A}$ in this case. It is also possible for some range of $m_{A} \lesssim 1 \mathrm{TeV}$ for LHC to access $b A \rightarrow b \mu^{+} \mu^{-}$production [105].

In figure 5, we show scatter plots of YUNS points with $R_{\mathrm{yuk}}<1.4$ and $\Delta<100$ in $a$ ) $m_{\tilde{t}_{1}}$ vs. $m_{\tilde{b}_{1}}$ and $\left.b\right) m_{\tilde{t}_{2}}$ vs. $m_{\tilde{b}_{2}}$ space. From frame $a$ ), we see as expected that $m_{\tilde{t}_{1}}$ and $m_{\tilde{b}_{1}}$ are correlated, with some solutions reaching well below $m_{\tilde{t}_{1}} \sim 500 \mathrm{GeV}$, which should be accessible to LHC searches. However, in this case these points all violate $B$-constraints, so 

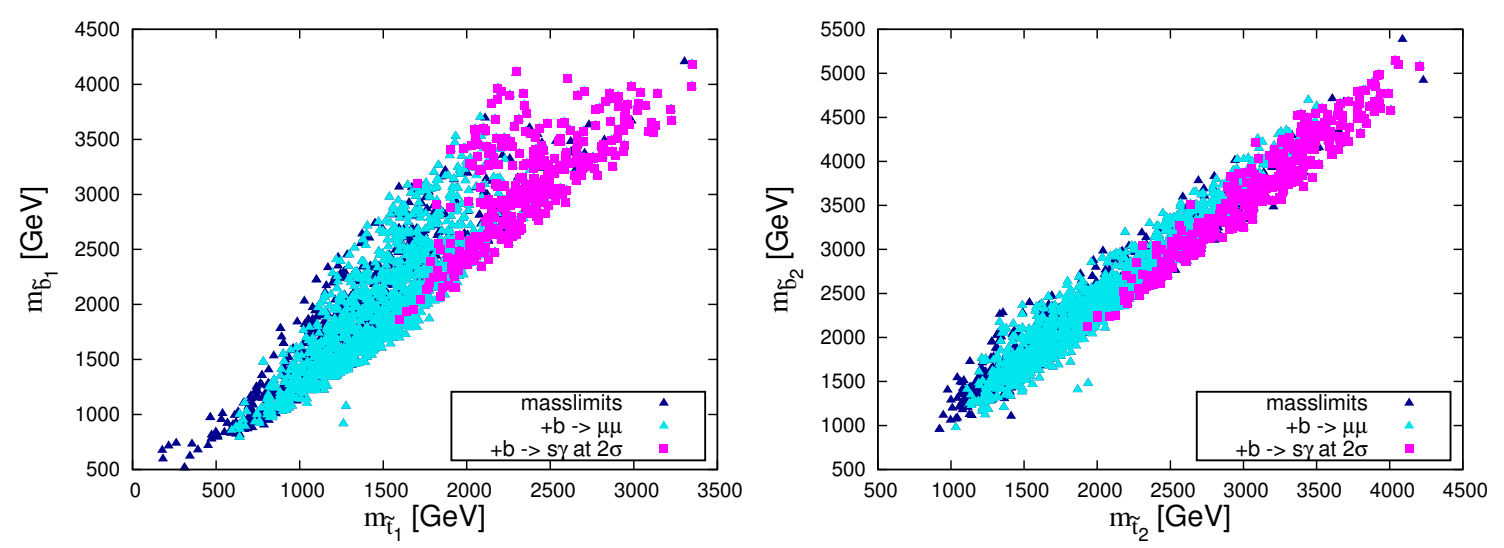

Figure 5. Distribution of scan points in a) $m_{\tilde{t}_{1}}$ vs. $m_{\tilde{b}_{1}}$ space and $\left.b\right) m_{\tilde{t}_{2}} v s . m_{\tilde{b}_{2}}$ space for $R_{\text {yuk }}<1.4$ and $\Delta<100$. Same color code as in figure 1 .

that requiring $B$-constraints within measured range requires instead $m_{\tilde{t}_{1}}, m_{\tilde{b}_{1}} \gtrsim 1.5 \mathrm{TeV}$, likely beyond the $14 \mathrm{TeV}$ LHC reach. In frame $b$ ), we see also that $m_{\tilde{b}_{2}}$ and $m_{\tilde{t}_{2}}$ are correlated. This is different from usual NS, where $m_{\tilde{b}_{2}}$ can be far above $m_{\tilde{t}_{1,2}}$ and $m_{\tilde{b}_{1}}$. The reason here is that $f_{b}$ is large and so there is also a non-negligible contribution to $\Sigma_{u}$ from $\tilde{b}_{1,2}$. Imposing $B$-constraints, we find $m_{\tilde{t}_{2}}$ and $m_{\tilde{b}_{2}}$ both $\gtrsim 2 \mathrm{TeV}$ (the former aids in lifting $m_{h}$ into its measured range).

\section{Benchmark points}

In this section we present several YUNS benchmark points, see table 1, and compare to one YUS benchmark point (HSb, from the "just-so" HS model) from ref. [90].

For HSb, $R_{\text {yuk }} \sim 1.02$, which is nearly perfect Yukawa coupling unification. The Higgs mass $m_{h} \simeq 127.8 \mathrm{GeV}$ is also sufficiently heavy. Unfortunately, the point is now excluded by LHC7 searches for multi-jet $+E_{T}^{\text {miss }}$ plus one $b$-tag searches, since $m_{\tilde{g}}$ is only $351 \mathrm{GeV}$. We also list in table 1 the EWFT measure; for HSb we have $\Delta=2489$, indicating exceptionally high level of fine-tuning. Much of this comes from the $\mu$ parameter which turns out to be of order $3 \mathrm{TeV}$, and thus requires a large value of $m_{H_{u}}^{2}$ at the weak scale to cancel against.

In contrast, point YUNS1 in column 3 has low fine-tuning of $\Delta=39$, at the cost of relaxing $R_{\text {yuk }}$ to 1.37 . For YUNS1, $m_{\tilde{g}} \simeq 1.3 \mathrm{TeV}$, with first/second generation squarks at $\sim 19 \mathrm{TeV}$. The point is likely beyond LHC8 reach, but should be accessible to LHC14 with $10-100 \mathrm{fb}^{-1}$. The point is slightly in violation of our listed condition on $B R(b \rightarrow s \gamma)$. However, by lowering $m_{0}(1,2)$ slightly, then $m_{\tilde{t}_{1}}$ is increased and also weak scale flavor violation is decreased $[103,104]$ (the GUT scale squark mass matrices become more nearly proportional to the identity matrix). For instance, lowering $m 0(1,2)$ to $15 \mathrm{TeV}$ increases $B R(b \rightarrow s \gamma)$ to $3.1 \times 10^{-4}$, while $R$ increases to 1.41 and $\Delta$ increases to 54 .

In column 4 , we list YUNS2 with $R_{\text {yuk }}=1.3$, as low as allowed by $B$-constraints, but with $\Delta \sim 100$. This point has $m_{\tilde{g}} \sim 4.3 \mathrm{TeV}$ and $m_{\tilde{q}} \sim 17.5 \mathrm{TeV}$, so it is likely beyond LHC reach, including a high-luminosity upgrade. While the higgsino-like chargino is only $112.5 \mathrm{GeV}$, it decays via 3-body mode into a higgsino-like $\widetilde{Z}_{1}$ with $m_{\widetilde{Z}_{1}}=106.2 \mathrm{GeV}$, so that 


\begin{tabular}{|lcccc|}
\hline parameter & HSb & YUNS1 & YUNS2 & YUNS3 \\
\hline$m_{16}(1,2)$ & 10000 & 19390.0 & 17149.4 & 19928.8 \\
$m_{16}(3)$ & 10000 & 5938.8 & 2490.4 & 2490.5 \\
$m_{1 / 2}$ & 43.9 & 444.4 & 1859.2 & 1809.1 \\
$A_{0}$ & -19947.3 & -6595.5 & -1374.1 & -319.1 \\
$\tan \beta$ & 50.398 & 52.09 & 54.2 & 51.7 \\
$\mu$ & 3132.6 & 136.0 & 106.1 & 169.6 \\
$m_{A}$ & 1825.9 & 884.1 & 541.2 & 776.4 \\
\hline$f_{t}$ & 0.557 & 0.578 & 0.563 & 0.549 \\
$f_{b}$ & 0.557 & 0.423 & 0.474 & 0.496 \\
$f_{\tau}$ & 0.571 & 0.561 & 0.618 & 0.590 \\
$R_{\text {yuk }}$ & 1.025 & 1.37 & 1.3 & 1.19 \\
$\Delta$ & 2489 & 39 & 105 & 48 \\
\hline$m_{\tilde{g}}$ & 351.2 & 1328.5 & 4309.5 & 4247.2 \\
$m_{\tilde{u}_{L}}$ & 9972.1 & 19396.8 & 17466.6 & 20189.0 \\
$m_{\tilde{t}_{1}}$ & 2756.5 & 1587.3 & 1598.0 & 509.3 \\
$m_{\tilde{b}_{1}}$ & 3377.1 & 2176.3 & 1857.1 & 793.1 \\
$m_{\tilde{e}_{R}}$ & 10094.7 & 19379.3 & 17153.2 & 19930.2 \\
$m_{\widetilde{W}_{1}}$ & 116.4 & 137.3 & 112.5 & 177.4 \\
$m_{\widetilde{Z}_{2}}$ & 113.8 & 147.4 & 112.0 & 176.2 \\
$m_{\widetilde{Z}_{1}}$ & 49.2 & 118.9 & 106.2 & 170.3 \\
$m_{h}$ & 127.8 & 123.1 & 123.8 & 123.3 \\
\hline $\mathrm{BR}\left(B \rightarrow X_{s} \gamma\right)$ & $3.1 \times 10^{-4}$ & $2.7 \times 10^{-4}$ & $2.8 \times 10^{-4}$ & $2.4 \times 10^{-4}$ \\
$\mathrm{BR}\left(B_{s} \rightarrow \mu^{+} \mu^{-}\right)$ & $8.1 \times 10^{-9}$ & $4.5 \times 10^{-9}$ & $4.6 \times 10^{-9}$ & $2.4 \times 10^{-8}$ \\
$\Omega_{\widetilde{Z}_{1}}^{\mathrm{TP}} h^{2}$ & 4613 & 0.01 & 0.004 & 0.007 \\
$\sigma^{\mathrm{SI}}\left(\widetilde{Z}_{1} p\right) \mathrm{pb}$ & $2.2 \times 10^{-13}$ & $4.5 \times 10^{-8}$ & $4.7 \times 10^{-9}$ & $2.9 \times 10^{-9}$ \\
$\sigma^{\mathrm{SD}}\left(\widetilde{Z}_{1} p\right) \mathrm{pb}$ & $1.2 \times 10^{-9}$ & $7.3 \times 10^{-4}$ & $3.6 \times 10^{-5}$ & $1.6 \times 10^{-5}$ \\
$\left.\langle\sigma v\rangle\right|_{v \rightarrow 0} \mathrm{~cm}{ }^{3} / \mathrm{s}$ & $1.9 \times 10^{-32}$ & $2.4 \times 10^{-25}$ & $3.3 \times 10^{-25}$ & $2.8 \times 10^{-25}$ \\
\hline
\end{tabular}

Table 1. Parameters and masses in GeV units for HSb [90] and three Yukawa-unified natural SUSY (YUNS) benchmark points. We also show $B$-decay constraints and dark matter relic density and (in)direct detection cross sections.

visible decay products are very soft, and likely impossible to observe above SM backgrounds at the LHC. Point YUNS3, listed in column 5, features $R_{\text {yuk }}$ as low as 1.19 , with $\Delta=48$. This point has $\operatorname{BR}\left(B \rightarrow X_{s} \gamma\right)=2.4 \times 10^{-4}$, and $\operatorname{BR}\left(B_{s} \rightarrow \mu^{+} \mu^{-}\right)=2.4 \times 10^{-8}$, so it falls out of the $B$-physics allowed range. While gluinos and first/second generation squarks 

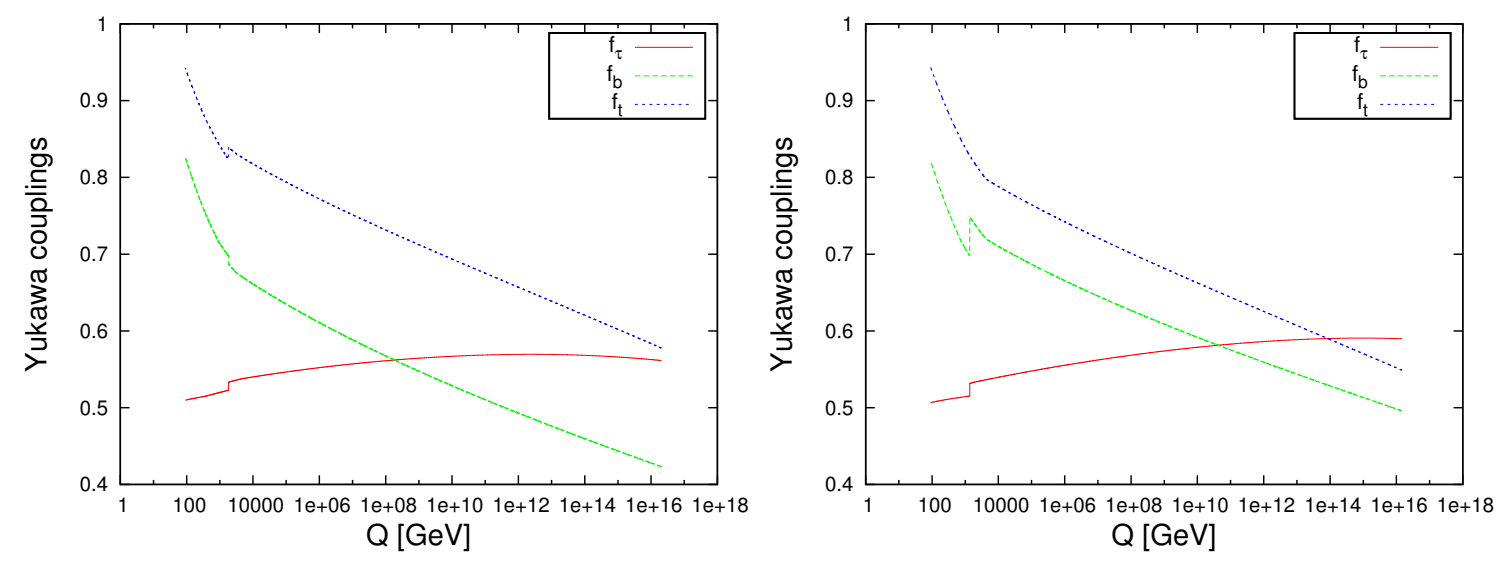

Figure 6. Evolution of Yukawa couplings for benchmark points YUNS1 (left) and YUNS3 (right) versus renormalization scale $Q$.

are beyond LHC reach, the rather light top and bottom squarks may be accessible to LHC searches. All these points have $\Omega_{\widetilde{Z}_{1}} h^{2} \ll 0.11$, leaving room for non-thermal higgsino production and axions. This contrasts point HSb, which has a much too thermal abundance and so would need an extremely light axino or huge late-time entropy production to tame this over-abundance [88].

In figure 6 , we show the Yukawa coupling evolution of $f_{t}, f_{b}$ and $f_{\tau}$ versus renormalization group scale $Q$, from $m_{\text {weak }}$ to $M_{\mathrm{GUT}}$ for benchmark points YUNS1 and YUNS3. These can be compared to similar plots for YUS, as in e.g. figure 6 of ref. [91]. The SUSY threshold corrections implemented at the scale $Q=\sqrt{m_{\tilde{t}_{1}} m_{\tilde{t}_{2}}}$ show up as jumps in the curves. In the case of YUS models, the $m_{b}$ threshold correction is positive due to a large $\tilde{t}_{i} \widetilde{W}_{j}$ loop contribution which goes like $\delta_{b} \sim\left(f_{t}^{2} / 32 \pi^{2}\right)\left(\mu A_{t} / m_{\tilde{t}}^{2}\right) \tan \beta$, where both $\mu$ and $A_{t}$ are extremely large. For YUNS models, with rather low $\mu$, these loops are suppressed and in the case of YUNS1, the $\tilde{g} \tilde{b}_{i}$ loops actually dominate, and are of opposite sign to the $\tilde{t}_{i} \widetilde{W}_{j}$ loops, leading to the slight downward jump of $f_{b}$ and thus bad Yukawa coupling unification. For YUNS3, the $\tilde{t}_{i} \widetilde{W}_{j}$ loops are larger, and the jump goes upwards, thus providing better Yukawa coupling unification.

\section{Yukawa-unified natural SUSY: LHC, ILC and DM searches}

In this section, we discuss the observable consequences of YUNS for LHC, ILC and direct and indirect WIMP and also axion searches.

\subsection{YUNS at LHC}

In natural SUSY models, it is favorable to have multi-TeV first/second generation squarks and sleptons because 1 . they are safely beyond current LHC searches, 2 . they provide at least a partial solution to the SUSY flavor and CP problems and 3. they provide additional suppression of third generation scalar masses via large 2-loop RGE effects. However, this means they are likely beyond any conceivable LHC reach. Third generation squarks may 
be much lighter, and in generic NS models are naively expected to be below the TeV scale (but see ref. [62] where 1-4 TeV third generation squarks work just fine, and lift the value of $m_{h}$ into its measured range). In the case of YUNS, with $\tan \beta \sim 50$, the combined light squarks and large $\tan \beta$ usually imply violation of $B$-constraints, and if these are imposed, then top and bottom squarks are beyond $1.5 \mathrm{TeV}$, and likely inaccessible to LHC searches.

For YUNS, the gluino mass may lie anywhere in the $1-5 \mathrm{TeV}$ range. It has been estimated in ref. [92-99] that LHC14 with $100 \mathrm{fb}^{-1}$ should be able to access gluino pair production in the case of heavy squarks for $m_{\tilde{g}}$ up to $1.8 \mathrm{TeV}$. In the case of YUNS with heavier top and bottom squarks, the $\tilde{g}$ is expected to dominantly decay via 3-body modes into $t \bar{t} \widetilde{Z}_{i}$ and $t b \widetilde{W}_{i}$ final states. The gluino pair events will thus contain multi-jets plus missing energy plus isolated leptons plus several identifiable $b$-jets [100]. While the higgsinolike chargino and neutralino production cross sections can be large, their decays to soft visible particles, arising from the small energy release in their 3-body decays, will be difficult to detect at LHC above SM backgrounds [78].

In the case where $m_{A} \lesssim 1 \mathrm{TeV}$, then it may be possible to detect $A, H \rightarrow \tau^{+} \tau^{-}$, especially if these are produced in association with $b$-jets, e.g. $p p \rightarrow b A, b H$ production. It may also be possible to detect $b A, b H$ production with $A, H \rightarrow \mu^{+} \mu^{-}$[105], since the production and decay are enhanced at large $\tan \beta$. In this case, the $A, H$ mass and width may be determined by reconstructing $m\left(\mu^{+} \mu^{-}\right)$. At large $\tan \beta$, this width is typically in the tends of $\mathrm{GeV}$ range and is very sensitive to $\tan \beta$. This reaction offers a method to distinguish YUNS from NS, in that the former is expected to occur at $\tan \beta \sim 50$.

\subsection{YUNS at ILC}

A linear $e^{+} e^{-}$collider operating at $\sqrt{s} \sim 250-500 \mathrm{GeV}$ would in many ways be an optimal discovery machine for YUNS. The reason is that by construction $\mu \lesssim 250 \mathrm{GeV}$, so chargino and neutralino pair production should always be available. While the small energy release

in $\widetilde{W}_{1}$ and $\widetilde{Z}_{2}$ decay is problematic at LHC, it should be much more easily observable in the clean environment of an $e^{+} e^{-}$collider. In this sense, an $e^{+} e^{-}$collider operating at $\sqrt{s} \sim 250-500 \mathrm{GeV}$ would be a higgsino in addition to a Higgs factory. It is also possible that some lighter third generation squarks are accessible to ILC with $\sqrt{s} \sim 1 \mathrm{TeV}$ or CLIC with $\sqrt{s}=3 \mathrm{TeV}$, depending if one avoids $B$-constraints and accepts the low mass, low $R_{\text {yuk }}$ solutions.

\subsection{Higgsino-like WIMPs}

A generic prediction of both NS and YUNS models is that the LSP is a higgsino-like WIMP with a typical under-abundance of thermally produced (TP) neutralinos $\Omega_{\widetilde{Z}_{1}}^{\mathrm{TP}} h^{2} \sim$ $0.002-0.01$. However, in cases where $m_{1 / 2}$ is as low as $\sim 300 \mathrm{GeV}$ and $\mu$ is as large as $200-$ $250 \mathrm{GeV}$, then there can be substantial bino-higgsino mixing, boosting $\Omega_{\widetilde{Z}_{1}}^{\mathrm{TP}} h^{2}$ up to 0.11 or even beyond. The situation is illustrated in figure 7 , where we plot $\Omega_{\widetilde{Z}_{1}}^{\mathrm{TP}} h^{2}$ versus $m_{\widetilde{Z}_{1}}$ and versus the $\widetilde{Z}_{1}$ higgsino fraction from YUNS models satisfying $R_{\text {yuk }}<1.4$ and $\Delta<100$.

The typical under-abundance is an appealing feature if one invokes the Peccei-Quinn solution to the strong CP problem, in which case one must introduce an axion superfield $\hat{a}$ 

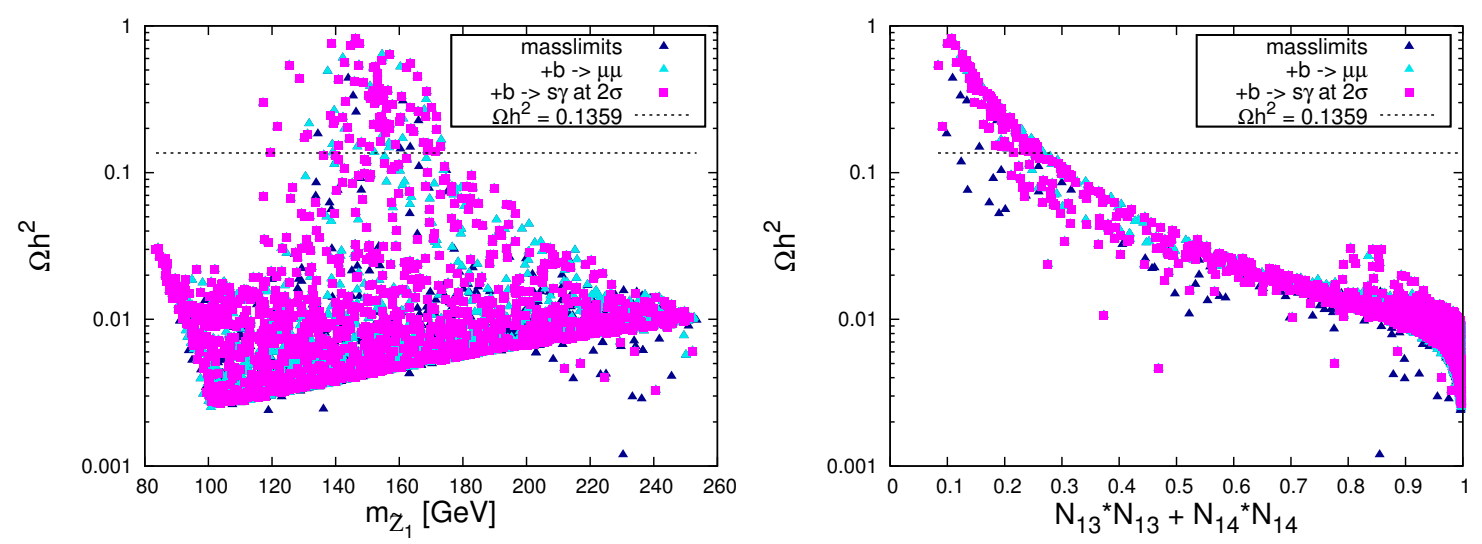

Figure 7. Thermal neutralino relic density $\Omega h^{2}$ versus $\widetilde{Z}_{1}$ mass (left) and versus $\widetilde{Z}_{1}$ higgsino fraction (right) for scan points with $R_{\text {yuk }}<1.4$ and $\Delta<100$. Same color code as in figure 1 .

which contains a pseudoscalar axion $a$ as well as a spin- $1 / 2$ axino $\tilde{a}$ and a spin-0 saxion $s$. In such models, one expects both saxion and axino masses at or around the SUSY breaking scale, so that dark matter is comprised of an axion-WIMP admixture. In this case, thermal production of axinos and subsequent decay to states such a $\tilde{g} g$ bolster the WIMP abundance beyond its TP-value. In addition, axions are produced as usual via coherent oscillations. It is also possible to suppress the WIMP abundance in the mixed $a \widetilde{Z}_{1}$ cosmology via latetime entropy production from saxion production and decay, although this case tends to be highly constrained by maintaining successful Big Bang nucleosynthesis (BBN). The upshot is that in the mixed $a \widetilde{Z}_{1}$ dark matter scenario, it may be possible to detect both a WIMP and an axion.

In the case of the YUNS model, the higgsino-like neutralinos have a substantial spinindependent (SI) direct detection cross section, as illustrated in table 1 , where $\sigma^{\mathrm{SI}}\left(\widetilde{Z}_{1} p\right) \sim$ $10^{-8} \mathrm{pb}$. While this level of direct detection cross section is now highly constrained by recent XENON100 [106] results, one must bear in mind that the higgsino-like WIMPs would constitute only a portion of the dark matter, so their local abundance might be up to a factor of 30 lower than is commonly assumed. In table 1 , we also list $\sigma^{\mathrm{SD}}\left(\widetilde{Z}_{1} p\right)$ (relevant for WIMP detection at IceCube) and $\left.\langle\sigma v\rangle\right|_{v \rightarrow 0}$, relevant for detection of dark matter annihilation into gamma rays or anti-matter throughout the cosmos. While these cross sections are also at potentially observable levels, again one must take into account that the overall WIMP abundance may be up to a factor of about 30 below what is commonly assumed. Plots of $\sigma^{\mathrm{SI}}\left(\widetilde{Z}_{1} p\right), \sigma^{\mathrm{SD}}\left(\widetilde{Z}_{1} p\right)$ and $\left.\langle\sigma v\rangle\right|_{v \rightarrow 0}$ have been presented in the case of higgsino-like WIMPs in refs. [78] and [81] and so similar plots will not be reproduced here.

\section{Summary and conclusions}

Previous analyses of $t-b-\tau$ Yukawa-unified models suffer from two problems: 1 . they tend to predict a light gluino $m_{\tilde{g}} \lesssim 500 \mathrm{GeV}$ (alhough solutions are possible for much heavier gluinos) which is now excluded by LHC searches, and 2. they suffer from extreme 
fine-tuning in the electroweak sector. In this paper, we examined how well the Yukawa couplings could unify in the natural SUSY context, where $\mu \sim 100-250 \mathrm{GeV}$, while at the same time requiring the light Higgs mass $m_{h} \sim 122-128 \mathrm{GeV}$. The small value of $\mu$ suppresses the large $\tilde{t}_{i} \widetilde{W}_{j}$ loop contributions to the $b$-quark Yukawa coupling which seem to be needed for precision Yukawa coupling unification. Nonetheless, by scanning over NUHM2 parameters with split third generation, we are able to find solutions with $R_{\text {yuk }}$ as low as 1.18. These solutions, with very light third generations squarks and $\tan \beta \sim 50$ tend to violate $B$-physics constraints. If $B$-physics constraints are imposed, then only $R_{\text {yuk }} \sim 1.3$ can be achieved.

The Yukawa-unified natural SUSY spectra have important differences from previous YU spectra. The gluino mass can easily be in the $1-4 \mathrm{TeV}$ range, thus avoiding LHC constraints from SUSY searches. The light higgsino-like charginos and neutralinos decay to soft particles, also avoiding LHC searches. However, light higgsinos should be easily accessible to an ILC with $\sqrt{s} \sim 0.25-05 \mathrm{TeV}$, as is typical of all NS models. In addition, as in all NS models, the lightest neutralino is higgsino-like with a typical thermal underabundance of WIMP dark matter. We regard this as a positive feature in that the WIMP abundance is typically increased in non-standard (but more attractive) cosmologies such as those conatining mixed axion-neutralino cold dark matter.

The question arises as to how to distinguish YUNS from ordinary NS. The YUNS model requires $\tan \beta \sim 50$, which leads to large production cross sections for heavy Higgs bosons $A$ and $H$ at LHC, and large widths for these particles. If the rare decays $A, H \rightarrow \mu^{+} \mu^{-}$ can be identified with suficiently high statistics (perhaps at a luminosity upgraded LHC), then the widths may be measured with precision, allowing one to highly constrain $\tan \beta$, and perhaps verify that it is consistent with YUNS models.

Note added. After completion of this study, a first measurement of $\operatorname{BR}\left(B_{s} \rightarrow \mu^{+} \mu^{-}\right)=$ $3.2_{-1.2}^{+1.5} \times 10^{-9}$ became available [107]. Requiring consistency with this measurement at the $2 \sigma$ level actually gives a less severe constraint than the upper bound in eq. (2.3). Our conclusions regarding the minimum achievable $R_{\text {yuk }}$ remain unchanged.

\section{Acknowledgments}

This work has been supported in part by the Office of Science, US Department of Energy and by IN2P3 under contract PICS FR-USA No. 5872. HB and SK acknowledge the hospitality and inspiring working atmosphere of the Aspen Center for Physics which is supported by the National Science Foundation Grant No. PHY-1066293.

Open Access. This article is distributed under the terms of the Creative Commons Attribution License which permits any use, distribution and reproduction in any medium, provided the original author(s) and source are credited. 


\section{References}

[1] H. Georgi, in Particles and Fields - 1974, C. Carlson eds., AIP press, New York U.S.A. (1974).

[2] H. Fritzsch and P. Minkowski, Unified Interactions of Leptons and Hadrons, Annals Phys. 93 (1975) 193 [INSPIRE].

[3] M. Gell-Mann, P. Ramond and R. Slansky, Color Embeddings, Charge Assignments and Proton Stability in Unified Gauge Theories, Rev. Mod. Phys. 50 (1978) 721 [InSPIRE].

[4] R. Mohapatra, Supersymmetric grand unification: An Update, hep-ph/9911272 [INSPIRE].

[5] S. Raby, Desperately seeking supersymmetry [susy], Rept. Prog. Phys. 67 (2004) 755 [hep-ph/0401155] [INSPIRE].

[6] E. Witten, Dynamical Breaking of Supersymmetry, Nucl. Phys. B 188 (1981) 513 [INSPIRE].

[7] R.K. Kaul, Gauge Hierarchy in a Supersymmetric Model, Phys. Lett. B 109 (1982) 19 [INSPIRE].

[8] S. Dimopoulos, S. Raby and F. Wilczek, Supersymmetry and the Scale of Unification, Phys. Rev. D 24 (1981) 1681 [inSPIRE].

[9] M. Einhorn and D. Jones, The Weak Mixing Angle and Unification Mass in Supersymmetric SU(5), Nucl. Phys. B 196 (1982) 475 [inSPIRE].

[10] W.J. Marciano and G. Senjanović, Predictions of Supersymmetric Grand Unified Theories, Phys. Rev. D 25 (1982) 3092 [inSPIRE].

[11] U. Amaldi, W. de Boer and H. Furstenau, Comparison of grand unified theories with electroweak and strong coupling constants measured at LEP, Phys. Lett. B 260 (1991) 447 [INSPIRE].

[12] J.R. Ellis, S. Kelley and D.V. Nanopoulos, Probing the desert using gauge coupling unification, Phys. Lett. B 260 (1991) 131 [inSPIRE].

[13] P. Langacker and M.-x. Luo, Implications of precision electroweak experiments for $M_{t}, \rho_{0}$, $\sin ^{2} \theta_{W}$ and grand unification, Phys. Rev. D 44 (1991) 817 [INSPIRE].

[14] B. Ananthanarayan, G. Lazarides and Q. Shafi, Top mass prediction from supersymmetric guts, Phys. Rev. D 44 (1991) 1613 [INSPIRE].

[15] B. Ananthanarayan, G. Lazarides and Q. Shafi, Radiative electroweak breaking and sparticle spectroscopy with $\tan \beta \simeq m_{t} / m_{b}$, Phys. Lett. B 300 (1993) 245 [INSPIRE].

[16] G.W. Anderson, S. Raby, S. Dimopoulos and L.J. Hall, Precise predictions for $m(t), V(c b)$ and tan Beta, Phys. Rev. D 47 (1993) 3702 [hep-ph/9209250] [inSPIRE].

[17] G. Anderson, S. Raby, S. Dimopoulos, L. Hall and G. Starkman, A Systematic SO(10) operator analysis for fermion masses, Phys. Rev. D 49 (1994) 3660 [hep-ph/9308333] [INSPIRE].

[18] V.D. Barger, M. Berger and P. Ohmann, The supersymmetric particle spectrum, Phys. Rev. D 49 (1994) 4908 [hep-ph/9311269] [InSPIRE].

[19] M.S. Carena, M. Olechowski, S. Pokorski and C. Wagner, Electroweak symmetry breaking and bottom-top Yukawa unification, Nucl. Phys. B 426 (1994) 269 [hep-ph/9402253] [INSPIRE]. 
[20] B. Ananthanarayan, Q. Shafi and X. Wang, Improved predictions for top quark, lightest supersymmetric particle and Higgs scalar masses, Phys. Rev. D 50 (1994) 5980 [hep-ph/9311225] [INSPIRE].

[21] R. Rattazzi and U. Sarid, The Unified minimal supersymmetric model with large Yukawa couplings, Phys. Rev. D 53 (1996) 1553 [hep-ph/9505428] [INSPIRE].

[22] T. Blazek, M.S. Carena, S. Raby and C.E. Wagner, A global $\chi^{2}$ analysis of electroweak data in $\mathrm{SO}(10)$ SUSY GUTs, Phys. Rev. D 56 (1997) 6919 [hep-ph/9611217] [INSPIRE].

[23] T. Blazek and S. Raby, Supersymmetric grand unified theories and global fits to low-energy data, Phys. Lett. B 392 (1997) 371 [hep-ph/9611319] [INSPIRE].

[24] T. Blazek and S. Raby, $b \rightarrow$ s $\gamma$ with large tan beta in MSSM analysis constrained by a realistic SO(10) model, Phys. Rev. D 59 (1999) 095002 [hep-ph/9712257] [INSPIRE].

[25] T. Blazek, S. Raby and K. Tobe, Neutrino oscillations in a predictive SUSY GUT, Phys. Rev. D 60 (1999) 113001 [hep-ph/9903340] [INSPIRE].

[26] T. Blazek, S. Raby and K. Tobe, Neutrino oscillations in an $\mathrm{SO}(10)$ SUSY GUT with $\mathrm{U}(2) \times \mathrm{U}(1)^{n}$ family symmetry, Phys. Rev. D 62 (2000) 055001 [hep-ph/9912482] [INSPIRE].

[27] S. Profumo, Neutralino dark matter, $b \tau$ Yukawa unification and nonuniversal sfermion masses, Phys. Rev. D 68 (2003) 015006 [hep-ph/0304071] [INSPIRE].

[28] C. Pallis, $b \tau$ unification with gaugino and sfermion mass nonuniversality, Nucl. Phys. B 678 (2004) 398 [hep-ph/0304047] [INSPIRE].

[29] M. Gomez, G. Lazarides and C. Pallis, Supersymmetric cold dark matter with Yukawa unification, Phys. Rev. D 61 (2000) 123512 [hep-ph/9907261] [InSPIRE].

[30] M. Gomez, G. Lazarides and C. Pallis, Yukawa quasi-unification, Nucl. Phys. B 638 (2002) 165 [hep-ph/0203131] [inSPIRE].

[31] M. Gomez, G. Lazarides and C. Pallis, On Yukawa quasiunification with $\mu$ less than 0 , Phys. Rev. D 67 (2003) 097701 [hep-ph/0301064] [INSPIRE].

[32] U. Chattopadhyay, A. Corsetti and P. Nath, Supersymmetric dark matter and Yukawa unification, Phys. Rev. D 66 (2002) 035003 [hep-ph/0201001] [INSPIRE].

[33] M.E. Gomez, T. Ibrahim, P. Nath and S. Skadhauge, WMAP dark matter constraints and Yukawa unification in SUGRA models with CP phases, Phys. Rev. D 72 (2005) 095008 [hep-ph/0506243] [INSPIRE].

[34] M. Badziak and K. Sakurai, LHC constraints on Yukawa unification in $\mathrm{SO}(10)$, JHEP 02 (2012) 125 [arXiv:1112.4796] [INSPIRE].

[35] M. Badziak, Yukawa unification in SUSY SO(10) in light of the LHC Higgs data, Mod. Phys. Lett. A 27 (2012) 1230020 [arXiv:1205.6232] [INSPIRE].

[36] A.S. Joshipura and K.M. Patel, Yukawa coupling unification in $\mathrm{SO}(10)$ with positive $\mu$ and a heavier gluino, Phys. Rev. D 86 (2012) 035019 [arXiv:1206.3910] [INSPIRE].

[37] I. Gogoladze, Q. Shafi and C.S. Un, $125 \mathrm{GeV}$ Higgs Boson from $t-b-\tau$ Yukawa Unification, JHEP 07 (2012) 055 [arXiv: 1203.6082] [INSPIRE].

[38] H. Baer, M.A. Diaz, J. Ferrandis and X. Tata, Sparticle mass spectra from $\mathrm{SO}(10)$ grand unified models with Yukawa coupling unification, Phys. Rev. D 61 (2000) 111701 [hep-ph/9907211] [INSPIRE]. 
[39] H. Baer et al., Yukawa unified supersymmetric $\mathrm{SO}(10)$ model: Cosmology, rare decays and collider searches, Phys. Rev. D 63 (2000) 015007 [hep-ph/0005027] [INSPIRE].

[40] H. Baer and J. Ferrandis, Supersymmetric $\mathrm{SO}(10)$ GUT models with Yukawa unification and a positive mu term, Phys. Rev. Lett. 87 (2001) 211803 [hep-ph/0106352] [INSPIRE].

[41] D. Auto et al., Yukawa coupling unification in supersymmetric models, JHEP 06 (2003) 023 [hep-ph/0302155] [INSPIRE].

[42] T. Blazek, R. Dermisek and S. Raby, Predictions for Higgs and supersymmetry spectra from $\mathrm{SO}(10)$ Yukawa unification with $\mu>0$, Phys. Rev. Lett. 88 (2002) 111804 [hep-ph/0107097] [INSPIRE].

[43] T. Blazek, R. Dermisek and S. Raby, Yukawa unification in $\mathrm{SO}(10)$, Phys. Rev. D 65 (2002) 115004 [hep-ph/0201081] [inSPIRE].

[44] R. Dermisek, S. Raby, L. Roszkowski and R. Ruiz De Austri, Dark matter and $B_{s} \rightarrow \mu^{+} \mu^{-}$ with minimal $\mathrm{SO}(10)$ soft SUSY breaking, JHEP 04 (2003) 037 [hep-ph/0304101] [INSPIRE].

[45] R. Dermisek, S. Raby, L. Roszkowski and R. Ruiz de Austri, Dark matter and $B_{s} \rightarrow \mu^{+} \mu^{-}$ with minimal SO(10) soft SUSY breaking II, JHEP 09 (2005) 029 [hep-ph/0507233] [INSPIRE].

[46] H. Baer, S. Kraml, S. Sekmen and H. Summy, Dark matter allowed scenarios for Yukawa-unified SO(10) SUSY GUTs, JHEP 03 (2008) 056 [arXiv:0801.1831] [INSPIRE].

[47] W. Altmannshofer, D. Guadagnoli, S. Raby and D.M. Straub, SUSY GUTs with Yukawa unification: A Go/no-go study using FCNC processes, Phys. Lett. B 668 (2008) 385 [arXiv:0801.4363] [INSPIRE].

[48] I. Gogoladze, R. Khalid, S. Raza and Q. Shafi, $t-b-\tau$ Yukawa unification for $\mu<0$ with a sub-TeV sparticle spectrum, JHEP 12 (2010) 055 [arXiv:1008.2765] [INSPIRE].

[49] I. Gogoladze, R. Khalid, S. Raza and Q. Shafi, Higgs and sparticle spectroscopy with gauge-Yukawa unification, JHEP 06 (2011) 117 [arXiv:1102.0013] [INSPIRE].

[50] G. Elor, L.J. Hall, D. Pinner and J.T. Ruderman, Yukawa unification and the superpartner mass scale, JHEP 10 (2012) 111 [arXiv:1206.5301] [INSPIRE].

[51] H. Baer, V. Barger and A. Mustafayev, Implications of a $125 \mathrm{GeV}$ Higgs scalar for LHC SUSY and neutralino dark matter searches, Phys. Rev. D 85 (2012) 075010 [arXiv:1112.3017] [INSPIRE].

[52] F. Brummer, S. Kraml and S. Kulkarni, Anatomy of maximal stop mixing in the MSSM, JHEP 08 (2012) 089 [arXiv:1204.5977] [InSPIRE].

[53] ATLAS collaboration, Observation of a new particle in the search for the Standard Model Higgs boson with the ATLAS detector at the LHC, Phys. Lett. B 716 (2012) 1 [arXiv: 1207.7214] [INSPIRE].

[54] CMS collaboration, Observation of a new boson at a mass of $125 \mathrm{GeV}$ with the CMS experiment at the LHC, Phys. Lett. B $\mathbf{7 1 6}$ (2012) 30 [arXiv:1207.7235] [INSPIRE].

[55] ATLAS collaboration, Search for supersymmetry in pp collisions at $\sqrt{s}=7 \mathrm{TeV}$ in final states with missing transverse momentum and $b^{-}$jets with the ATLAS detector, Phys. Rev. D 85 (2012) 112006 [arXiv:1203.6193] [INSPIRE]. 
[56] H. Baer, S. Raza and Q. Shafi, A Heavier gluino from $t-b-\tau$ Yukawa-unified SUSY, Phys. Lett. B 712 (2012) 250 [arXiv:1201.5668] [INSPIRE].

[57] H. Baer, V. Barger, P. Huang, A. Mustafayev and X. Tata, Radiative natural SUSY with a 125 GeV Higgs boson, Phys. Rev. Lett. 109 (2012) 161802 [arXiv:1207.3343] [INSPIRE].

[58] R. Kitano and Y. Nomura, A solution to the supersymmetric fine-tuning problem within the MSSM, Phys. Lett. B 631 (2005) 58 [hep-ph/0509039] [INSPIRE].

[59] R. Kitano and Y. Nomura, Supersymmetry, naturalness and signatures at the LHC, Phys. Rev. D 73 (2006) 095004 [hep-ph/0602096] [INSPIRE].

[60] R. Barbieri and G. Giudice, Upper Bounds on Supersymmetric Particle Masses, Nucl. Phys. B 306 (1988) 63 [InSPIRE].

[61] D.M. Ghilencea, H.M. Lee and M. Park, Tuning supersymmetric models at the LHC: A comparative analysis at two-loop level, JHEP 07 (2012) 046 [arXiv: 1203.0569] [INSPIRE].

[62] C. Wymant, Optimising Stop Naturalness, arXiv:1208.1737 [INSPIRE].

[63] S. Antusch, L. Calibbi, V. Maurer, M. Monaco and M. Spinrath, Naturalness and GUT Scale Yukawa Coupling Ratios in the CMSSM, Phys. Rev. D 85 (2012) 035025 [arXiv:1111.6547] [INSPIRE].

[64] S. Antusch, L. Calibbi, V. Maurer, M. Monaco and M. Spinrath, Naturalness of the Non-Universal MSSM in the Light of the Recent Higgs Results, arXiv:1207.7236 [INSPIRE].

[65] G. Giudice and A. Masiero, A Natural Solution to the mu Problem in Supergravity Theories, Phys. Lett. B 206 (1988) 480 [INSPIRE].

[66] F.E. Paige, S.D. Protopopescu, H. Baer and X. Tata, ISAJET 7.69: A Monte Carlo event generator for $p p, \bar{p} p$ and $e^{+} e^{-}$reactions, hep-ph/0312045 [INSPIRE].

[67] H. Baer, C.-H. Chen, R.B. Munroe, F.E. Paige and X. Tata, Multichannel search for minimal supergravity at $p \bar{p}$ and $e^{+} e^{-}$colliders, Phys. Rev. D 51 (1995) 1046 [hep-ph/9408265] [INSPIRE].

[68] H. Baer, J. Ferrandis, S. Kraml and W. Porod, Treatment of threshold effects in supersymmetric spectrum computations, Phys. Rev. D 73 (2006) 015010.

[69] S.P. Martin and M.T. Vaughn, Two loop renormalization group equations for soft supersymmetry breaking couplings, Phys. Rev. D 50 (1994) 2282 [Erratum ibid. D 78 (2008) 039903] [hep-ph/9311340] [INSPIRE].

[70] H.E. Haber and R. Hempfling, The Renormalization group improved Higgs sector of the minimal supersymmetric model, Phys. Rev. D 48 (1993) 4280 [hep-ph/9307201] [INSPIRE].

[71] R. Hempfling, Yukawa coupling unification with supersymmetric threshold corrections, Phys. Rev. D 49 (1994) 6168 [inSPIRE].

[72] L.J. Hall, R. Rattazzi and U. Sarid, The Top quark mass in supersymmetric $\mathrm{SO}(10)$ unification, Phys. Rev. D 50 (1994) 7048 [hep-ph/9306309] [INSPIRE].

[73] M.S. Carena, M. Olechowski, S. Pokorski and C. Wagner, Electroweak symmetry breaking and bottom-top Yukawa unification, Nucl. Phys. B 426 (1994) 269 [hep-ph/9402253] [INSPIRE].

[74] D.M. Pierce, J.A. Bagger, K.T. Matchev and R.-j. Zhang, Precision corrections in the minimal supersymmetric standard model, Nucl. Phys. B 491 (1997) 3 [hep-ph/9606211] [INSPIRE]. 
[75] K.L. Chan, U. Chattopadhyay and P. Nath, Naturalness, weak scale supersymmetry and the prospect for the observation of supersymmetry at the Tevatron and at the CERN LHC, Phys. Rev. D 58 (1998) 096004 [hep-ph/9710473] [INSPIRE].

[76] H. Baer, S. Kraml, A. Lessa, S. Sekmen and X. Tata, Effective supersymmetry at the LHC, JHEP 10 (2010) 018 [arXiv:1007.3897] [InSPIRE].

[77] M. Asano, H.D. Kim, R. Kitano and Y. Shimizu, Natural supersymmetry at the LHC, JHEP 12 (2010) 019 [arXiv:1010.0692] [INSPIRE].

[78] H. Baer, V. Barger and P. Huang, Hidden SUSY at the LHC: the light higgsino-world scenario and the role of a lepton collider, JHEP 11 (2011) 031 [arXiv:1107.5581] [INSPIRE].

[79] M. Papucci, J.T. Ruderman and A. Weiler, Natural SUSY endures, JHEP 09 (2012) 035 [arXiv:1110.6926] [INSPIRE].

[80] C. Brust, A. Katz, S. Lawrence and R. Sundrum, SUSY, the third generation and the LHC, JHEP 03 (2012) 103 [arXiv:1110.6670] [INSPIRE].

[81] H. Baer, V. Barger, P. Huang and X. Tata, Natural supersymmetry: LHC, dark matter and ILC searches, JHEP 05 (2012) 109 [arXiv:1203.5539] [INSPIRE].

[82] ATLAS collaboration, Search for squarks and gluinos with the ATLAS detector in final states with jets and missing transverse momentum using $4.7 \mathrm{fb}^{-1}$ of $\sqrt{\mathrm{s}}=7 \mathrm{Te} \mathrm{V}$ proton-proton collision data, arXiv:1208.0949 [INSPIRE].

[83] CMS collaboration, Search for supersymmetry in hadronic final states using MT2 in pp collisions at $\sqrt{s}=7 \mathrm{TeV}$, JHEP 10 (2012) 018 [arXiv:1207.1798] [INSPIRE].

[84] CMS collaboration, Search for neutral Higgs bosons decaying to $\tau$ pairs in pp collisions at $\sqrt{s}=7$ TeV, Phys. Lett. B 713 (2012) 68 [arXiv:1202.4083] [INSPIRE].

[85] Joint LeP 2 Supersymmetry Working Group, Combined LEP Chargino Results up to $208 \mathrm{GeV}$, http://lepsusy.web.cern.ch/lepsusy/www/inos_moriond01/charginos_pub.html.

[86] M. Misiak et al., Estimate of $B(\bar{B} \rightarrow X(s) \gamma)$ at $O\left(\alpha_{s}^{2}\right)$, Phys. Rev. Lett. $98(2007) 022002$ [hep-ph/0609232] [INSPIRE].

[87] A. Crivellin et al., SUSY_FLAVOR v2: A Computational tool for FCNC and CP-violating processes in the MSSM, arXiv:1203.5023 [INSPIRE].

[88] H. Baer, A. Lessa and W. Sreethawong, Coupled Boltzmann calculation of mixed axion/neutralino cold dark matter production in the early universe, JCAP 01 (2012) 036 [arXiv:1110.2491] [INSPIRE].

[89] M. Albrecht, W. Altmannshofer, A.J. Buras, D. Guadagnoli and D.M. Straub, Challenging $\mathrm{SO}(10)$ SUSY GUTs with family symmetries through FCNC processes, JHEP 10 (2007) 055 [arXiv:0707.3954] [INSPIRE].

[90] H. Baer, M. Haider, S. Kraml, S. Sekmen and H. Summy, Cosmological consequences of Yukawa-unified SUSY with mixed axion/axino cold and warm dark matter, JCAP 02 (2009) 002 [arXiv:0812.2693] [INSPIRE].

[91] H. Baer, S. Kraml and S. Sekmen, Is 'just-so' Higgs splitting needed for $t-b-\tau$ Yukawa unified SUSY GUTs?, JHEP 09 (2009) 005 [arXiv:0908.0134] [INSPIRE].

[92] H. Baer, X. Tata and J. Woodside, Multi-lepton signals from supersymmetry at hadron super colliders, Phys. Rev. D 45 (1992) 142 [INSPIRE]. 
[93] H. Baer, C.-h. Chen, F. Paige and X. Tata, Signals for minimal supergravity at the CERN large hadron collider: Multi-jet plus missing energy channel, Phys. Rev. D 52 (1995) 2746 [hep-ph/9503271] [INSPIRE].

[94] H. Baer, C.-h. Chen, F. Paige and X. Tata, Signals for minimal supergravity at the CERN large hadron collider. 2: Multi-lepton channels, Phys. Rev. D 53 (1996) 6241 [hep-ph/9512383] [inSPIRE].

[95] H. Baer, C.-h. Chen, M. Drees, F. Paige and X. Tata, Probing minimal supergravity at the CERN LHC for large $\tan \beta$, Phys. Rev. D 59 (1999) 055014 [hep-ph/9809223] [INSPIRE].

[96] H. Baer, C. Balázs, A. Belyaev, T. Krupovnickas and X. Tata, Updated reach of the CERN $L H C$ and constraints from relic density, $b \rightarrow s \gamma$ and $a(\mu)$ in the mSUGRA model, JHEP 06 (2003) 054 [hep-ph/0304303] [INSPIRE].

[97] S. Abdullin and F. Charles, Search for SUSY in (leptons +) jets + E (T)(miss) final states, Nucl. Phys. B 547 (1999) 60 [hep-ph/9811402] [INSPIRE].

[98] CMS collaboration, Discovery potential for supersymmetry in CMS, J. Phys. G 28 (2002) 469 [hep-ph/9806366] [INSPIRE].

[99] B. Allanach, J. Hetherington, A. Parker and B. Webber, Naturalness reach of the large hadron collider in minimal supergravity, JHEP 08 (2000) 017.

[100] H. Baer, X. Tata and J. Woodside, Phenomenology of gluino decays via loops and top quark Yukawa coupling, Phys. Rev. D 42 (1990) 1568 [inSPIRE].

[101] F. Gabbiani, E. Gabrielli, A. Masiero and L. Silvestrini, A Complete analysis of FCNC and $C P$ constraints in general SUSY extensions of the standard model,

Nucl. Phys. B 477 (1996) 321 [hep-ph/9604387] [INSPIRE].

[102] S. Bertolini, F. Borzumati, A. Masiero and G. Ridolfi, Effects of supergravity induced electroweak breaking on rare B decays and mixings, Nucl. Phys. B 353 (1991) 591 [INSPIRE].

[103] H. Baer and M. Brhlik, QCD improved $b \rightarrow s \gamma$ constraints on the minimal supergravity model, Phys. Rev. D 55 (1997) 3201 [hep-ph/9610224] [INSPIRE].

[104] H. Baer, M. Brhlik, D. Castano and X. Tata, $b \rightarrow s \gamma$ constraints on the minimal supergravity model with large $\tan \beta$, Phys. Rev. D 58 (1998) 015007 [hep-ph/9712305] [INSPIRE].

[105] H. Baer, A. Belyaev, C. Kao and P. Svantesson, Exploring neutralino dark matter resonance annihilation via $b A, b H \rightarrow b \mu^{+} \mu^{-}$at the LHC, Phys. Rev. D 84 (2011) 095029 [arXiv:1106.5055] [INSPIRE].

[106] XENON100 collaboration, E. Aprile et al., Dark Matter Results from 225 Live Days of XENON100 Data, Phys. Rev. Lett. 109 (2012) 181301 [arXiv:1207.5988] [INSPIRE].

[107] LHCB collaboration, R. Aaij et al., First evidence for the decay $B_{s} \rightarrow \mu^{+} \mu^{-}$, arXiv:1211.2674 [INSPIRE]. 